\title{
Targeted Data Acquisition for Improved Reproducibility and Robustness of Proteomic Mass Spectrometry Assays
}

\author{
Mikhail M. Savitski, Frank Fischer, Toby Mathieson, Gavain Sweetman, \\ Manja Lang, and Marcus Bantscheff \\ Department of Analytical Sciences, Cellzome AG, Heidelberg, Germany
}

Quantitative mass spectrometry-based proteomic assays often suffer from a lack of robustness and reproducibility. We here describe a targeted mass spectrometric data acquisition strategy for affinity enriched subproteomes-in our case the kinome-that enables a substantially improved reproducibility of detection, and improved quantification via isobaric tags. Inclusion mass lists containing $\mathrm{m} / \mathrm{z}$, charge state, and retention time were created based on a set of 80 shotgun-type experiments performed under identical experimental conditions. For each target protein, peptides were selected according to their frequency of observation and isobaric tag for relative and absolute quantitation (iTRAQ) reporter ion quality. Retention times of selected peptides were aligned using similarity driven pairwise alignment strategy yielding $<1 \mathrm{~min}$ standard deviation for $4 \mathrm{~h}$ gradients. Multiple fragmentation of the same peptides resulted in better statistics and more precise reporter ion based quantification without any loss in coverage. Overall, $24 \%$ more target proteins were quantified using the targeted data acquisition approach, and precision of quantification improved by $>1.5$-fold. We also show that a combination of higher energy collisional dissociation (HCD) with collisional induced dissociation (CID) outperformed pulsed-Q-dissociation (PQD) on the OrbitrapXL. With the CID/ HCD based targeted data acquisition approach 10\% more quantifiable target proteins were identified and a 2-fold increase in quantification precision was achieved. We have observed excellent reproducibility between different instruments, underlining the robustness of the approach. (J Am Soc Mass Spectrom 2010, 21, 1668-1679) (C) 2010 American Society for Mass Spectrometry

$\mathrm{W}$ hen combined with mass spectrometry, affinity enrichment techniques enable the detailed quantitative study of a defined subproteome. For example, protein complexes can be studied using either immunoprecipitation or affinity purification using genetically engineered bait proteins (e.g., TAP tagged proteins). Moreover, selective enrichment of post-translationally modified proteins can be achieved, e.g., ubiquitinated [1] or tyrosine phosphorylated [2] proteins. When pharmacologically active small molecule compounds are immobilized on a solid support, these affinity matrices can be employed to characterize the protein target profile of these compounds directly from cell or tissue extracts, making this chemical proteomics approach a powerful tool for mode-of action studies of, e.g., candidate drug molecules (for recent reviews see: [3-5]). Recently, a variety of chemical proteomics technologies have emerged that enable the selective enrichment of enzyme classes, directly from any cell line or tissue [6-10]. Modern quantita-

Address reprint requests to Drs. M. Savitski and M. Bantscheff, Department of Analytical Sciences, Cellzome AG Meyerhofstrasse 1, 69117 Heidelberg, Germany. E-mail: mikhail.savitski@cellzome.com and marcus.bantscheff@ cellzome.com tive mass spectrometry techniques allow to study how cellular perturbations like growth factor stimulation or drug treatment impact the affinity captured subproteomes [11].

Recently, several approaches to selectively enrich for a large fraction of the human kinome using immobilized nonselective kinase inhibitors or acyl phosphatenucleotide analogues were reported [6, 8-10]. A particularly relevant application of these target class selective enrichment techniques utilizing the conserved ATP binding pocket is their application in competition binding experiments with ATP competitive kinase inhibitor compounds. In a previous report, we described a proteomic assay utilizing mixed kinase inhibitor beads ("kinobeads") for kinome wide profiling of cellular targets of kinase inhibitor molecules [6]. In a competition binding assay, binding potencies of ATP-competitive inhibitor compounds were determined by measuring relative amounts of affinity-captured kinases as a function of inhibitor concentration using isobaric tagging for relative and absolute quantitation (iTRAQ) and mass spectrometry.

Here, we report that reproducibility of the proteomic target profiles and precision of protein quantification is 
substantially improved by a targeted acquisition strategy. Based on a dataset of 80 shotgun experiments, we selected the most suitable peptides for each kinase likely to be detected under the experimental conditions. Selection criteria included the frequency of observation and the quality of the iTRAQ reporter ion signal information obtained. We obtained $24 \%$ higher kinase coverage and $>1.5$-fold higher precision by using the targeted data acquisition approach. Additionally, we combined the targeted approach with the implementation of higher energy dissociation (HCD) [12] on the Orbitrap in combination with collisional induced dissociation (CID), which gave an additional $10 \%$ gain in kinase coverage and a 2 -fold gain in precision. We concur that the inclusion mass list-driven targeted data acquisition approach of isobarically labeled peptides will be advantageous for a variety of affinity purification based mass spectrometry assays.

\section{Methods}

\section{Sample Preparation}

Kinase enrichment using mixed kinase inhibitor resins were performed as described previously in [6]. Briefly, a 1:1 mixture of Jurkat and Ramos cell lysates was used at a final protein concentration of $5 \mathrm{mg} / \mathrm{mL}$; 1 - $\mathrm{mL}$ lysate aliquots were incubated with $35-\mu \mathrm{L}$ kinobead slurry for $1 \mathrm{~h}$, and bound kinases were eluted after washing using $50-\mu \mathrm{L} \times 2$ SDS sample buffer. Samples were run into a denaturing gel for $20 \mathrm{~min}$ to remove reagents incompatible with tryptic digestion. Tryptic in-gel digestion and iTRAQ labeling enrichment were done as described in [6]. Briefly, reduced and carbamidomethylated kinobead eluates were concentrated on $4 \%-12 \% \quad \nu$-PAGE gels (Invitrogen, Carlsbad, CA, USA) by running sample $\sim 1 \mathrm{~cm}$ into the gel to remove reagents incompatible with tryptic digestion and iTRAQ labeling. After staining with colloidal Coomassie, gel lanes were cut into three slices and subjected to in-gel digestion. Subsequently, peptide extracts were labeled with iTRAQ reagents (Applied Biosystems, Foster City, CA, USA) by adding $10-\mu \mathrm{L}$ reagent in ethanol and incubation for $1 \mathrm{~h}$ at $20{ }^{\circ} \mathrm{C}$ in $60 \%$ ethanol, $40 \mathrm{mM}$ triethylammoniumbicarbonate, $\mathrm{pH}$ 8.53. After quenching of the reaction with glycine, all labeled extracts of one-gel lane were combined and mixed with differently labeled extracts.

Reproducibility analyses were performed from a pooled sample of several competition binding experiments using the described kinase enrichment procedure [6], and displaying various degrees of displacement for individual kinases. This sample is referred to as the "reference sample" in the remainder of the text.

\section{Reference Data for Creation of Inclusion Lists}

Results from 80 experiments performed using various compounds for competition and a 1:1 mixture of Jurkat and Ramos cell lysates were used to create an initial inclusion list. Peptide data from all experiments including reporter ion signal intensities were stored in a relational database. Parameters that were stored with each peptide include: Mascot score, experimental retention time, modifications, charge state, ion accumulation time for the MS/MS spectrum, and reporter ion areas.

\section{Mass Spectrometry}

Experiments were performed on Thermo LTQ Orbitrap XL and Thermo LTQ Orbitrap Velos [13] mass spectrometers that were coupled to split-less Eksigent NanoLCs (Dublin, CA, USA). Peptides were separated on a custom made $50 \mathrm{~cm} \times 75 \mathrm{uM}$ (ID) reversed-phase column (Reprosil, Maisch, Germany) and gradient elution was performed from $2 \%$ acetonitrile to $40 \%$ acetonitrile in $0.1 \%$ formic acid within $4 \mathrm{~h}$ using a flow rate of $250 \mathrm{~nL} / \mathrm{min}$. The Orbitrap XL mass spectrometer was operated using the XCalibur Developers kit 2.0.7. The Orbitrap Velos mass spectrometer was operated using the XCalibur Developers kit 2.1.0. Intact peptides were detected in the Orbitraps at 60,000 resolution. Internal calibration was performed using the ion signal of $\left(\mathrm{Si}\left(\mathrm{CH}_{3}\right)_{2} \mathrm{O}\right)_{6} \mathrm{H}^{+}$at $m / z 445.120025$ as a lock mass [14]. Maximal ion accumulation time allowed on the LTQ Orbitraps was $1 \mathrm{~s}$ for MS; automatic gain control was used to prevent over-filling of the ion trap. No FT preview scan was used. The minimum MS signal for triggering MS/MS was set to 1000. PQD settings were optimized as described in Bantscheff et al. [15]. The normalized collision energy was set to $29 \%, Q$ value was 0.55 , and an activation time of $0.34 \mathrm{~ms}$ was used.

HCD settings. Target value FT: 5E4 ions, collision energy: 70\%, maximum FT fill time $500 \mathrm{~ms}$, isolation width: 2.5 Da. Fragment ions were detected in the Orbitrap at a resolution of 7500 . CID settings: Target value LTQ: 1e4 ions, max LTQ fill time $100 \mathrm{~ms}$, collision energy: $35 \%$, isolation width $2.5 \mathrm{Da}$.

For shotgun experiments up to six PQD sequencing events on the top six peaks were allowed. Monoisotopic precursor selection was enabled. Non-peptide monoisotopic recognition was not enabled. Charge state rejection was enabled. Unassigned charge states and charge state 1 were rejected. Repeat count was set to 1 . Exclusion mass width low was set to $1 \mathrm{Da}$ and exclusion mass width high to $2 \mathrm{Da}$. Repeat duration was set to $30 \mathrm{~s}$, exclusion duration was set to $120 \mathrm{~s}$.

Targeted data acquisition. The created inclusion mass lists were saved in a CSV format, with separate columns for MS Mass $(\mathrm{m} / \mathrm{z})$, Start (min), End (min), and MS Charge State. Note: The MS charge state information in the inclusion lists can only be used when the charge recognition function is switched on. The assigned charge state of the precursor has then to match the charge state on the inclusion list to be fragmented. The $m / z$ values were in column one, Start values in column two, End values in column three. The files were im- 
ported into the global parent mass list. Up to $6 \mathrm{PQD}$ only (or four pairs of CID/HCD) sequencing events on the top six peaks (or top four peaks) matching to $\mathrm{m} / \mathrm{z}$ and retention time windows on the inclusion list were allowed. Monoisotopic precursor selection was enabled. Non-peptide monoisotopic recognition was not enabled. Charge state rejection was enabled. Unassigned charge states and charge state 1 were rejected. Repeat count was set to 4 . Exclusion mass width low was set to $7.5 \mathrm{ppm}$ and exclusion mass width high to $7.5 \mathrm{ppm}$. Repeat duration was set to $30 \mathrm{~s}$; exclusion duration was set to $120 \mathrm{~s}$. The use of global parent and reject lists was enabled. The parent mass width was set to $7.5 \mathrm{ppm}$ for both high and low $\mathrm{m} / \mathrm{z}$.

\section{Protein Identification}

The acquired raw data were processed with in-house developed software. MGF files were created and submitted to the Mascot search engine [16]. The following settings were used: $7.5 \mathrm{ppm}$ precursor mass accuracy (monoisotopic mass), 0.8 Da fragment ion mass accuracy. The following modifications were selected, variable modifications: oxidation (methionine), iTRAQ (N-term), acetyl (protein $\mathrm{N}$-term), fixed modifications: iTRAQ (lysine), carbamidomethyl (cysteine). The instrument type was chosen as ESI-TRAP, the enzyme specificity as Trypsin/P, and the maximum number of missed cleavages was set to 3 . The data were searched against an in-house curated version of the human International Protein Index database combined with a decoy version thereof $[17,18]$. Our database contains a total of 163,476 protein sequences ( $50 \%$ forward, $50 \%$ reverse) and represents a nonredundant composite of International Protein Index versions 1.0-3.54. It is further supplemented with protein sequences of bovine serum albumin, porcine trypsin, and mouse, rat, and sheep keratins. Protein identification acceptance criteria for kinase enriched samples were solely based on spectrum to peptide sequence assignments that represented the best match (bold red definition of Mascot) and had a 10× higher Mascot probability than the second best match. For protein identifications with only one single peptide meeting these criteria, we required the Mascot score to be at least 33. Experiments with a decoy database indicate a $<1 \%$ false discovery rate for protein identification. For identifications where multiple peptides met these criteria, the decoy search results indicated $<0.1 \%$ false discovery rate on protein level.

\section{Protein Quantification}

Centroided iTRAQ reporter ion signals (114.111 Da, 115.108 Da, $116.112 \mathrm{Da}, 117.115 \mathrm{Da}$ ) were computed by the XCalibur software operating the mass spectrometer and extracted from MS data files using in-house developed software. Only spectra of peptides unique for identified proteins were used for relative protein quantification. iTRAQ reporter ion intensities were multi- plied with the ion accumulation time yielding an area value proportional to the number of reporter ions present in the ion trap [15]. Fold changes indicating relative abundancies were calculated using a linear model in which the iTRAQ intensities of the individual peptides of two conditions are plotted on the $x$ and $y$ axis. A straight line, forced to go through the origin, was fitted to the data. The slope of the line was the measure for the determined fold change. For each protein, 3-fold changes were calculated (114 to 117, 115 to 117 , and 116 to 117 ) [15].

For the CID/HCD setup, the iTRAQ reporter ion signals were extracted from the HCD spectrum and linked to the corresponding CID spectrum performed on the same precursor. The fold change ratio calculation was then performed as described above.

\section{Signal to Interference Calculation}

Precursor abundance (PAb) was extracted from the MS scan performed before the MS/MS event and is the sum of the integrated ion intensities over the isotope cluster. Precursor interference at the time of the MS/MS event was calculated as PAb divided by the sum of all ion signals observed within the isolation window (summed up with a weighting of 1 in the \pm 1 Da region of the ${ }^{12} \mathrm{C}$ peak of the precursor and a weighting of 0.5 in the regions +1 to $1.5 \mathrm{Da}$ and -1 to $-1.5 \mathrm{Da}$ ). Consequently, values close to one indicate little and values close to 0 a high degree of interference caused by co-eluting components.

\section{Automated RT Alignment}

The RT alignment is performed in two iterations. During the first iteration, the last experiment $E_{1}$ that has been performed on the instrument is aligned in turn to all other experiments in the database. The alignment quality is estimated for each experiment. Before the second iteration the experiments in the database are ordered according to decreasing alignment quality. The $E_{1}$ experiment is then aligned to each experiment in the database again and the retention times, peptide combinations that are present in the database experiment, but not in the $\mathrm{E}_{1}$ experiment, are added after each alignment.

\section{First Iteration}

The alignment procedure uses the last experiment, $\mathrm{E}_{1}$ that was performed on the instrument with the current LC setup, as a starting point (alignment seed). For each peptide (unique sequence, modification combination) that has a Mascot score of above 20, the retention time corresponding to the earliest sequencing event of this peptide is selected, $R T_{-} E_{1}=\left\{\left(\right.\right.$ pep $\left._{1}, \mathrm{rt}_{1}\right),\left(\right.$ pep $\left._{2}, \mathrm{rt}_{2}\right), \ldots$, $\left(\right.$ pep $\left.\left._{\mathrm{L}}, \mathrm{rt}_{\mathrm{L}}\right)\right\}$, pep $_{n}$ are the distinct peptides, $\mathrm{rt}_{n}$ are their retention times divided by the gradient time, and $\mathrm{L}$ is 
the total number of distinct peptides with Mascot score $>20$ in $\mathrm{E}_{1}$.

Next the peptide, $\mathrm{RT}_{-} \mathrm{E}_{1}$ data is compared with an experiment in the database, $E_{2}$. The RT_E $E_{2}$ data is selected for $E_{2}$ using the same criteria as for $E_{1}$. The retention times that point to the same peptide in $\mathrm{E}_{1}$ and $\mathrm{E}_{2}$ are paired, $\mathrm{RT} \mathrm{E}_{1 \_} \mathrm{E}_{2}=\left\{\left(\mathrm{rt}_{1 \_} \mathrm{e} 2, \mathrm{rt}_{1 \_} \mathrm{e} 1\right)\right.$, $\left.\left(\mathrm{rt}_{2} \mathrm{e} 2, \mathrm{rt}_{2 \_} \mathrm{e} 1\right), \ldots,\left(\mathrm{rt}_{\mathrm{M}-} \mathrm{e} 2, \mathrm{rt}_{\mathrm{M}-} \mathrm{e} 1\right)\right\}$ where $\mathrm{M}$ is the number of common peptides. If $\mathrm{M}>100$, an alignment is performed. This is done by a least-squares fitting of a fourth order polynomial, $\mathrm{P} 4$ to the $\mathrm{M}$ ( $\left.\mathrm{rt}_{n-} \mathrm{e} 2, \mathrm{rt}_{n-} \mathrm{e} 1\right)$ pairs, where $\mathrm{rt}_{n \_} \mathrm{e} 2$ comprise the $\mathrm{x}$ coordinates and $\mathrm{rt}_{n \_} \mathrm{e} 1$ the $\mathrm{y}$ coordinates. The aligned retention time values are obtained by transforming the $\mathrm{rt}_{n-} \mathrm{e} 2$ values with P4, obtaining the $\mathrm{rt}_{n-}$ e2_P4 values. Subsequently, the retention time difference vector is calculated, diffV = [rt_e1- rt_e2_P4, rt_e1-rt_e2_P4,..., rt_e1-rt_e2_P4], the standard deviation of the retention time differences, StD(diffV) and the median, Med(diffV). Then an outlier removal step is performed. All rt_e2_P4 values that deviate by more than $3 * \operatorname{StD}($ diffV) from Med(diffV) are removed. The alignment is performed again without the outliers and the recalculated $\operatorname{StD}(\operatorname{diffV})$ value is assigned to $\mathrm{E}_{2}$.

The data from $E_{1}$ is compared in this way to all the $\mathrm{N}-1$ experiments, ( $\mathrm{N}$ total number of experiments), in the database. In the end $\mathrm{N}-1 \mathrm{StD}($ diffV) values are calculated corresponding to N-1 experiments.

\section{Second Iteration}

The N-1 experiments are ordered according to increasing $\mathrm{StD}$ (diffV) values, $\left\{\mathrm{E}_{2}, \mathrm{E}_{3}, \ldots, \mathrm{E}_{\mathrm{N}-1}\right\} \rightarrow\left\{\mathrm{E}_{2^{\prime}}, \mathrm{E}_{3^{\prime}}, \ldots\right.$, $\left.E_{N-1^{\prime}}\right\}$. The data from $E_{2^{\prime}}$ is aligned to $E_{1}$, the outliers removed and the data realigned again as in the first iteration. Now the retention times of the peptides with Mascot score above 20 that are present in $\mathrm{E}_{2}$, but not in $\mathrm{E}_{1}$ are transformed using the fourth order polynomial from the second alignment and added to the RT_ $\mathrm{E}_{1}$ data, RT_E $E_{1}=\left\{\left(\right.\right.$ pep $\left._{1}, \mathrm{rt}_{1}\right),\left(\right.$ pep $\left._{2}, \mathrm{rt}_{2}\right), \ldots,\left(\right.$ pep $\left._{\mathrm{L}}, \mathrm{rt}_{\mathrm{L}}\right)$, $\left.\left(\operatorname{pep}_{\mathrm{L}+1}, \mathrm{rt}_{\mathrm{L}+1}\right), \ldots,\left(\operatorname{pep}_{\mathrm{L}+\mathrm{K}}, \mathrm{rt}_{\mathrm{L}+\mathrm{K}}\right)\right\}$, where $\mathrm{K}$ is the number of added peptide, aligned retention time combinations from $\mathrm{E}_{2^{\prime}}$.

This is repeated for all N-1 experiments. In the last step the retention times for peptides that are used for the inclusion list are multiplied with the gradient time of the planned experiment and matched to the corresponding $m / z$ values. The Start and End times are calculated by subtracting and adding the desired time window, $t$ _w from all the retention times on the list, with the minimum allowed value being 0 , and maximum allowed value being the gradient time.

\section{Results and Discussion}

In a previous report, we described a proteomic assay utilizing mixed kinase inhibitor resins beads ("kinobeads") for kinome wide profiling of cellular targets of kinase inhibitor molecules [6]. In a competition binding assay binding potencies of ATP-competitive inhibitor compounds were determined by measuring relative amounts of affinity-captured precipitated kinases as function of inhibitor concentration using isobaric peptide tagging (iTRAQ) and quantitative mass spectrometry (iTRAQ) (Figure 1a). Affinities of inhibitor compounds against $>100$ kinases were determined in one LC-MS/MS run using pulsed-Q-dissociation (PQD) on an Orbitrap mass spectrometer operated in shotgun mode. However, comparison of results obtained for larger panels of inhibitor molecules is impaired by the limited reproducibility of target protein identifications using the shotgun approach (Figure 1b). High mass accuracy inclusion lists using high-resolution mass spectrometers have recently been reported as an alternative to SRM-based strategies for targeted data acquisition $[19,20]$ and can be readily combined with isobaric labeling methods for quantitative mass spectrometry.

To determine the most suitable peptides per kinase, results from 80 experiments performed under standardized experimental conditions and analyzed on Orbitrap XL mass spectrometers using PQD in shotgun mode were uploaded into a relational database (Figure 2). Peptide retention times were aligned using a two-stage similarity driven polynomial alignment strategy (see Materials and Methods section). For each detected protein, only peptides were considered that were identified with a Mascot score $>20$, and were fully iTRAQ labeled (N-terminus and lys residues), and were unique for the protein (sequence not shared with other proteins). For each target protein detected peptides were sorted according to (frequency of detection)*(summed reporter ion area). Peptides detected in several charge states in the dataset were treated as multiple entities. The peptides were sorted in descending order according to this criterion, and the top 10 peptides were selected for each protein. For these peptides inclusion mass lists containing $m / z$, charge state and retention time were generated and uploaded to the instrument acquisition software before data acquisition. For each targeted data acquisition (TDA) experiment, a consistency check was performed between the aligned retention times on the inclusion list and the experimental retention times. If a significant shift ( $>5 \mathrm{~min}$ ), or a non linear perturbation had occurred, the retention times were realigned starting with the latest experiment as the alignment seed and the inclusion list was updated.

\section{Alignment of Retention Times}

The two stage retention time alignment is used because we have noticed that experiments performed on analytical separation columns of different lengths (e.g., $20 \mathrm{~cm}$ versus $50 \mathrm{~cm}$ ) or using slightly different gradients have a non-negligible difference in the order of which the peptides elute. Hence one would first like to align all experiments done with the same type of column, and then the experiments done with other set ups. The ordering of alignments according 

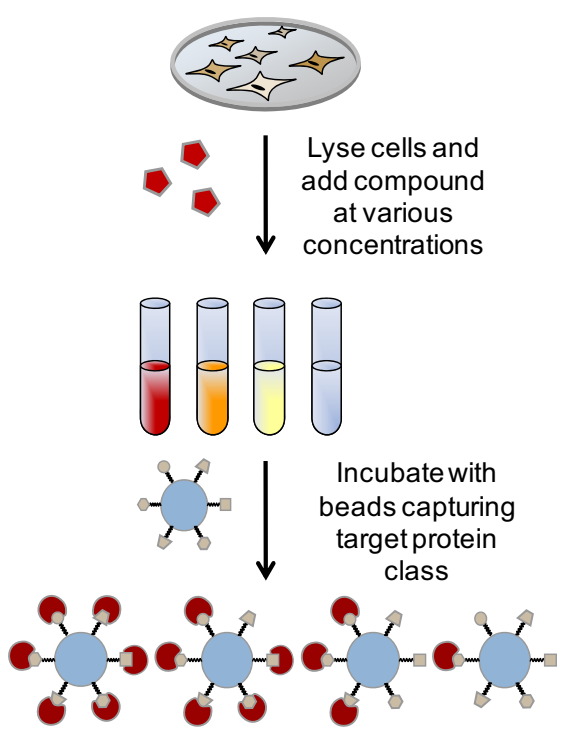

Measure influence of inhibitor on capturing
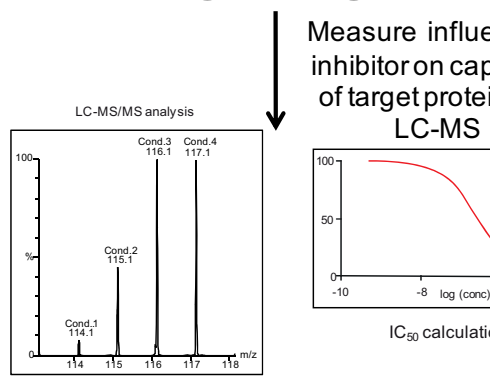

Figure 1. (a) Schematic representation of a kinase inhibitor profiling experiment using target class selective enrichment. The compound of interest is allowed to bind to its target proteins in a cellular extract. Then beads containing immobilized unspecific kinase inhibitors are added. Kinases with affinity to the test compound are occupied in their ATP binding site, hence a dose dependent reduction of binding of these kinases to the beads will be observed. Quantitative mass spectrometry enables determination of $\mathrm{IC}_{50} \mathrm{~s}$. In contrast, equal amounts of kinases for which the test compound has no affinity are detected. (b) Automated data dependent acquisition leads to limited reproducibility of identified kinases between LC-MS runs. This impairs comparison of selectivity profiles of a larger panel of test compounds (X: not detected). The goal is to reduce the number of 'not detected' kinases using TDA.

to the alignment quality between experiments takes care of this automatically.

An example comparing aligned retention times with measured retention times is shown in the left part of Figure 2. The green lines represent the applied retention time window of $\pm 15 \mathrm{~min}$. The standard deviation of the time differences between the aligned and measured retention times is $1 \mathrm{~min}$, which is a typical value obtained using the applied retention time alignment strategy. The average time shift is $-0.74 \mathrm{~min}$. We have observed that the average time shift from experiment to experiment has a standard deviation of 2-3 min for a standard $4 \mathrm{~h}$ gradient provided that no changes to the LC system are made between experiments. Hence the precision (typical standard deviation of $1 \mathrm{~min}$ ) of the aligned retention time is better than the accuracy (average shift of 2-3 $\mathrm{min}$ ). As a consequence, whenever practical considerations do not allow updating of inclusion lists for each run (e.g., when several runs are to be performed unattended) it is advisable to use wider retention time windows (15-30 $\mathrm{min})$ on the inclusion lists.

\section{Specificity of the Accurate Mass for Precursor Selection}

Given the high mass accuracy, $7.5 \mathrm{ppm}$, used for matching the $\mathrm{m} / \mathrm{z}$ from the inclusion list to the precursor ion and the medium complexity of affinity purified samples ( $<600$ detectable proteins) it is tempting to speculate that the mass accuracy alone without the retention time window should be sufficient to specifically target the peptides of interest. However, we found that even when applying a retention time window of 15 min the $7.5 \mathrm{ppm}$ accuracy requirement is not enough to exclusively single out the precursors of interest. In a TDA experiment with 1906 peptides on the inclusion list and $\mathrm{a} \pm 15 \mathrm{~min}$ retention time window 1074 peptides were identified from the list. However, apart from the performed MS/MS spectra pointing to the targeted 


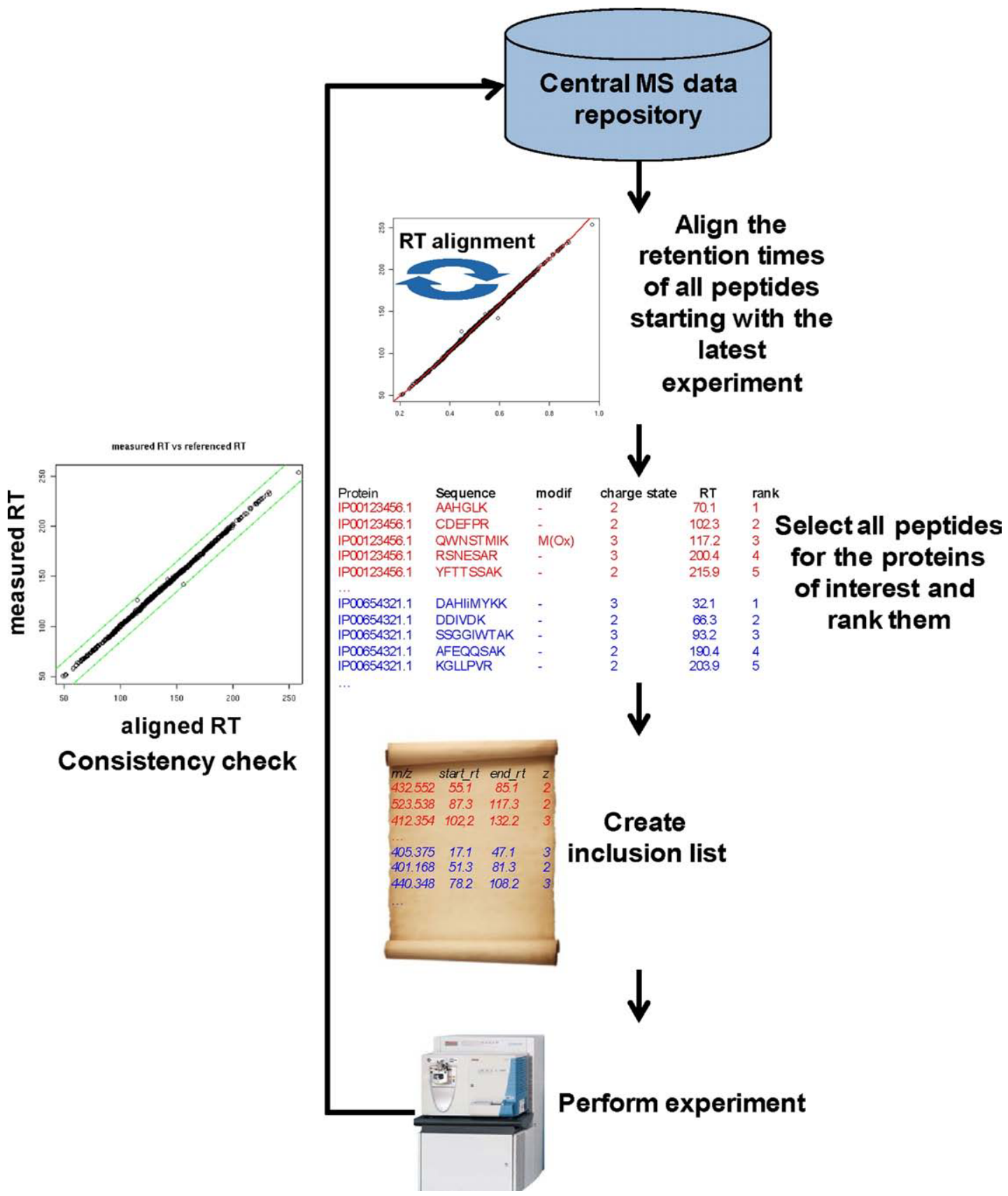

Figure 2. Scheme for the targeted data acquisition approach. The retention times of all peptides are aligned against each other. The peptides detected for the proteins of interest are ranked according to their frequency of detection and summed reporter ion area. The top 10 peptides for each protein are selected for the inclusion list. A targeted MS experiment is performed. The retention times of the acquired data are checked for consistency, plot on the left hand side, green lines: retention time window $= \pm 15 \mathrm{~min}$, s.d. $=1 \mathrm{~min}, 4 \mathrm{~h}$ gradient, and the data is imported into the MS data repository.

1074 chromatographic peaks, an additional set of tandem mass spectra was performed pointing to another 161 chromatographic peaks. $M / z$ values determined for these additional species all were within $7.5 \mathrm{ppm}$ of the $\mathrm{m} / \mathrm{z}$ of the identified inclusion list peptides and retention times were all within 15 min from the respective inclusion list peptides. These additional MS/MS spectra either did not lead to any reliable peptide identifications, or resulted from and led to identification of peptides that were not on the inclusion list. Furthermore, in 10 cases peptides from the inclusion list were identified less than $15 \mathrm{~min}$ apart in retention time and with $\mathrm{m} / \mathrm{z}$ differences of less than $7.5 \mathrm{ppm}$. In a repeat experiment with a retention time window of $30 \mathrm{~min}$, 1016 peptides (58 less than in the 15 min window experiment) were identified and MS/MS spectra pointing to 199 (38 more than in 15 min window experiment) chromatographic peaks that did not belong to peptides 
on the inclusion list were performed. This indicates that sensitivity and selectivity of high mass accuracy inclusion list experiments depend to a certain degree on the inclusion mass retention time window that can be achieved in day-to-day operation.

A particularly difficult case is shown in Figure 3a. Here two inclusion list peptides are identified, ATNM(Ox)EFAVK, from RSK2 kinase, and ATNMEYAVK, from RSK3 kinase. The two peptides have an identical elemental composition and, hence, identical $\mathrm{m} / \mathrm{z}$ values. The retention time difference is less than four minutes. This example illustrates that to detect as many peptides as possible, a short dynamic exclusion time is required. An accurate mass tag (AMT) [21, 22] strategy for identifying two isomers spaced by four minutes would have to rely on a retention time alignment with a standard deviation of $1 \mathrm{~min}$ to distinguish the two species with a $99 \%$ confidence.

\section{Reproducibility of Peptide Identifications}

Seven repeat experiments in shotgun and TDA mode were performed on the reference sample using pulsedQ-dissociation. For TDA experiments, the inclusion mass list contained 1534 peptides corresponding to 155 kinases. In total, 762 inclusion list peptides were detected in shotgun mode and 961 peptides were detected in targeted analysis mode representing $49.7 \%$ and $62.7 \%$ of the inclusion list. Comparison of the generated tandem mass spectra with the inclusion list showed that in TDA mode the instrument almost exclusively (in $99.97 \%$ of the cases) selected precursors whose $m / z$ were on the list and within the specified retention time windows. For each detected inclusion list peptide frequency of occurrence was calculated; 587 inclusion list peptides were detected in seven out of seven experiments in TDA mode compared with 206 in shotgun mode (Figure $3 b$ ). Overall, the average peptide frequency in shotgun experiments was 0.50 while for TDA experiments the average peptide frequency was 0.82 . The peptide reproducibility achieved within a TDA experiment and the difference in reproducibility between TDA and shotgun experiments are both dependent on sample complexity. The difference between shotgun and TDA would probably be even higher if a more complex sample would be analyzed. For lower complexity samples the reproducibility for TDA would most likely have been higher.

The increase in peptide reproducibility corroborates the observations of Jaffe et al. [20]. In this study, the authors were able to detect peptides in plasma that were difficult to detect with shotgun using a targeted approach. In our experiments, typically $50 \%$ to $60 \%$ of the inclusion list peptides were identified using the targeted analysis approach. This relatively low number might be attributed to two main factors. (1) To fully explore the sensitivity gain possible with targeted data acquisition, we included target proteins on the list, for which chances of detection were very low (i.e., that were detected only once in the 80 shotgun experiments used to set up the inclusion list). (2) Whenever 10 or fewer different peptides were detected for a specific target protein in the reference dataset, we included all peptides ever detected on the inclusion list, although their frequency of occurrence in the reference dataset might have been very low.

\section{Multiple Fragmentation of Target Peptides}

Multiple fragmentation of peptides by using high repeat count settings is usually omitted in shotgun experiments because it is detrimental to protein coverage. To illustrate this, we performed two shotgun experiments back-to-back on a kinase enriched sample. We observed a $20 \%$ drop in kinase identifications when the experiment was performed with a repeat count of four as compared with one, and quite remarkably a drop of $27 \%$ when only considering kinases quantified with at least 4 spectra. In a typical shotgun run (repeat count 1) 8500 spectra were acquired identifying 4082 different peptides. Since for targeted data acquisition only $\sim 1500$ different peptides were to be monitored, we explored
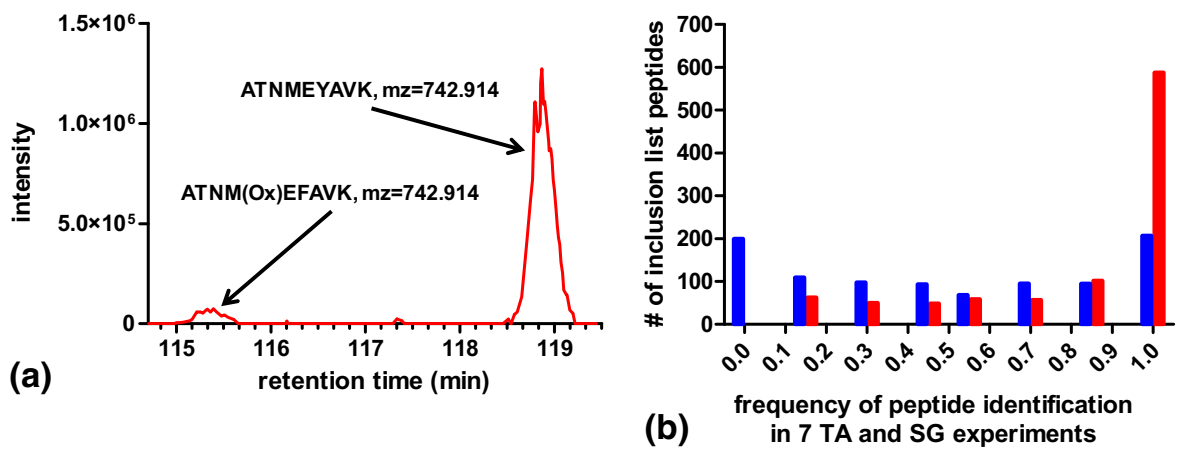

Figure 3. (a) Elution profiles of two inclusion list peptides with different sequences, but identical elemental compositions. (b) The frequency of inclusion list peptides occurrence in seven shotgun (blue bars) and seven targeted analysis (red bars) experiments is calculated as (\# of experiments in which the peptide was observed)/7. The statistics are done for peptides that were seen at least once in either the seven shotgun or seven targeted analysis experiments. 
the use the free instrument acquisition time for performing multiple fragmentations on the same precursor to improve quantification of peptides on the inclusion list.

In fact, when up to four repeat analyses per inclusion list peptide were allowed for TDA experiments, on average 30\% fewer spectra were acquired in the targeted analysis experiments (average number of spectra, 5711) than in shotgun experiments (average number of spectra, 8160). The additional repeats also required less instrument time than an average MS/MS spectrum acquired in shotgun mode because the repeat fragmentation events take place higher up the eluting chromatographic peak (Figure 4a). On average, the first sequencing event was taking place $10 \mathrm{~s}$ before the apex of the chromatographic peak, the second sequencing event $7 \mathrm{~s}$ before, the third, $3 \mathrm{~s}$ before, and the fourth took place $1 \mathrm{~s}$ after the apex. Hence, less ion accumulation time was required to achieve the target ion value in the ion trap. Figure $4 \mathrm{~b}$ exemplifies this for one of the seven TDA experiments. The median ion accumulation time value for the first repeat was $218 \mathrm{~ms}$, almost identical to the median acquisition time for an MS/MS for one of the seven shotgun experiments, $215 \mathrm{~ms}$. The median of the second repeat was 179 ms, of the third 137 $\mathrm{ms}$, and $119 \mathrm{~ms}$ for the fourth.

Co-fragmentation (fragmentation of other ions along with the precursor ion of interest) affects the quantifi- cation precision in iTRAQ based quantitative experiments [23, 24]. To get an estimate on how much co-fragmentation occurs for each peptide we developed the signal-to-interference score (s2i). S2i is defined as the ratio of the precursor ion intensities divided by the total signal measured within the isolation window applied (for details see the Methods section). A higher s2i value translates to higher quantification precision. This was evaluated by comparing two replicate TDA experiments. In these, fold changes of peptides identified from precursors with an $\mathrm{s} 2 \mathrm{i}$ score of $>0.8$ reproduced with an $R^{2}$ value of 0.69 . While the fold changes of peptides from precursors with an s2i score of $<0.8$ reproduced with a much inferior $R^{2}$ value of 0.40 . This clearly indicates that the $\mathrm{s} 2 \mathrm{i}$ value is a good measure for assessing the quantitation quality of a peptide.

The s2i was calculated for the precursors that were sequenced four times and led to a reliable identification of an inclusion list peptide (Figure $4 \mathrm{c}$ and $\mathrm{d}$ ). The first sequencing event that was performed at the start of the chromatographic peak had a low average s $2 \mathrm{i}$ of 0.571 , indicating substantial co-fragmentation. For the subsequent sequencing events increasingly higher signal-tointerference (i.e., less co-fragmentation) values were determined, going up to an average value of 0.756 for the sequencing event corresponding to the fourth repeat count. Hence, signal interference should be substantially reduced for samples acquired using targeted data

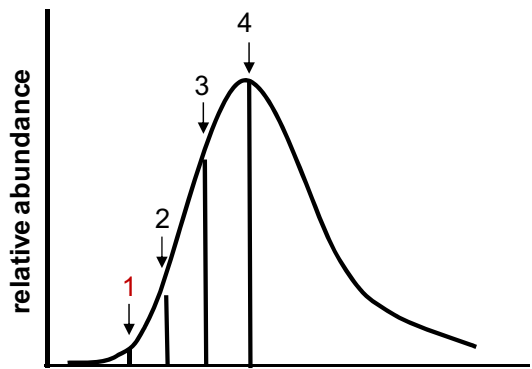

(a)

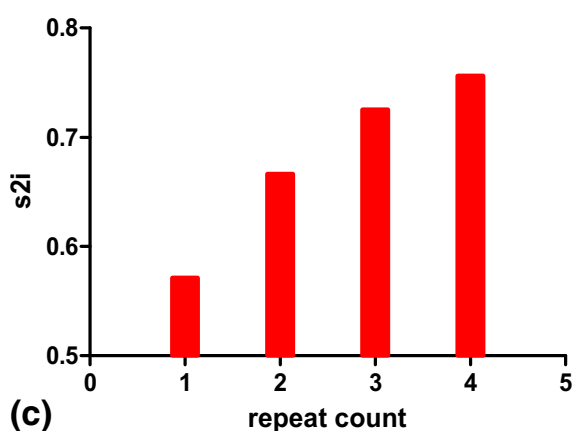

(c)

repeat count
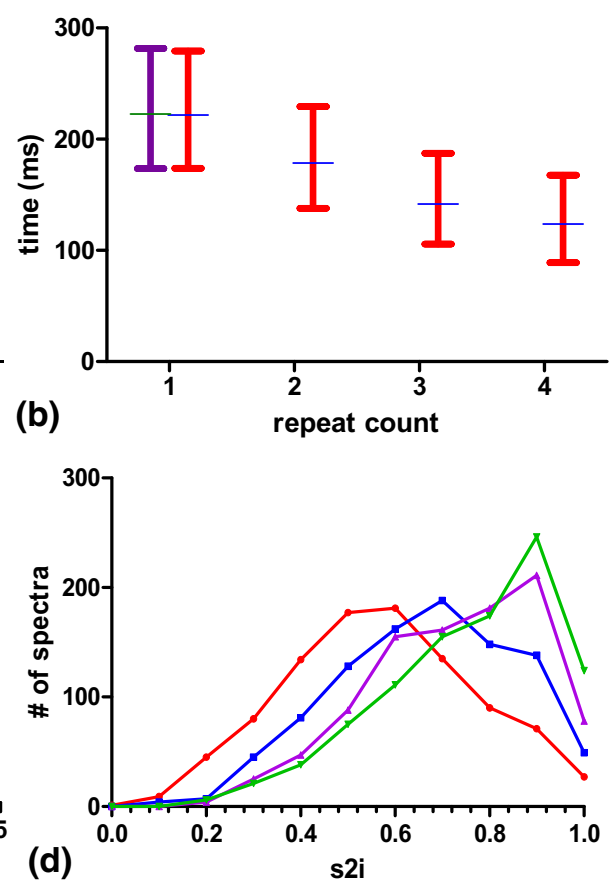

Figure 4. (a) Locations of repeat sequencing events across the chromatographic peak in a targeted analysis experiment. (b) Median ion acquisition time, and 25\%-75\% time intervals for shotgun experiment, blue marker (median) with violet interval $(25 \%-75 \%$ range), and for different repeat counts in a targeted analysis experiment, blue markers with red intervals. (c) Average signal to interference for different repeat counts in a targeted analysis experiment. (d) Distributions of signal to interferences for precursors selected at different repeat counts, red for first repeat, blue for second repeat, violet for third repeat, and green for fourth repeat. 
acquisition and multiple repeat counts compared with the shotgun approach where all sequencing events behave like those corresponding to the first repeat count. Note however that not all sequencing events corresponding to the first repeat have low s2i. Out of the 661 cases where a precursor was fragmented four times (approximately $70 \%$ of all identified inclusion list peptides), in 310 cases the fourth repeat had the highest s2i, in 105 cases the third repeat, in 54 cases the second, and in 37 cases the first, indicating that even though statistically the s2i is on average at its highest at the top of the chromatographic peak, there will be a significant number of cases where there is a low s2i near the top of the peak due to co-eluting fragments, but a high s2i at the beginning of the peak due to the absence of coelution. This again demonstrates the benefit of having multiple sequencing events across the chromatographic peak.

\section{Target Protein Coverage and Precision of Quantification}

Comparison of identified kinases for the seven shotgun replicate analyses and the seven TDA experiments indicated higher coverage and reproducibility using the targeted data acquisition approach. The average number of identified kinases increased by 8.7 kinases using the targeted approach (116 SG, $124.7 \mathrm{TA}$ ) with a stan- dard error for shotgun being 0.8 and for targeted analysis 0.7 . The average number of kinases quantified by at least four spectra increased by 28.7 kinases, from shotgun: 89.7 to 118.4 with targeted analysis (standard error for shotgun of 1.3 and for targeted analysis of 0.9 ). The number of kinases reproduced in all seven experiments with at least four spectra was also higher in targeted analysis than in shotgun, 104 versus 80 kinases (see supplementary Tables 1 and 2, which can be found in the electronic version of this article). The large increase of kinases quantified from four or more spectra is partly because a 4 repeat count setting was used. The average number of inclusion list peptides identified in TDA experiments was 809.4 with a standard error of 5.2.

Next, we calculated and compared the fold changes of the identified kinases in two repeat experiments (two for shotgun and two for TDA analyses) using the PQD setup on the same instrument, to assess the precision of quantification in the three approaches. One hundred seven kinases were identified in both shotgun experiments with at least one assigned spectrum. Comparison of individual relative abundancies determined for each kinase revealed a standard deviation of the $\log _{2}$ ratios of the relative changes of 0.282 (Figure $5 b$ ) and an $R^{2}$ value of 0.50 (Figure $5 \mathrm{c}$ ). In particular, the relative changes determined for kinases quantified with few spectra $(<4$ spectra) were poorly reproduced. In contrast, quantifi-
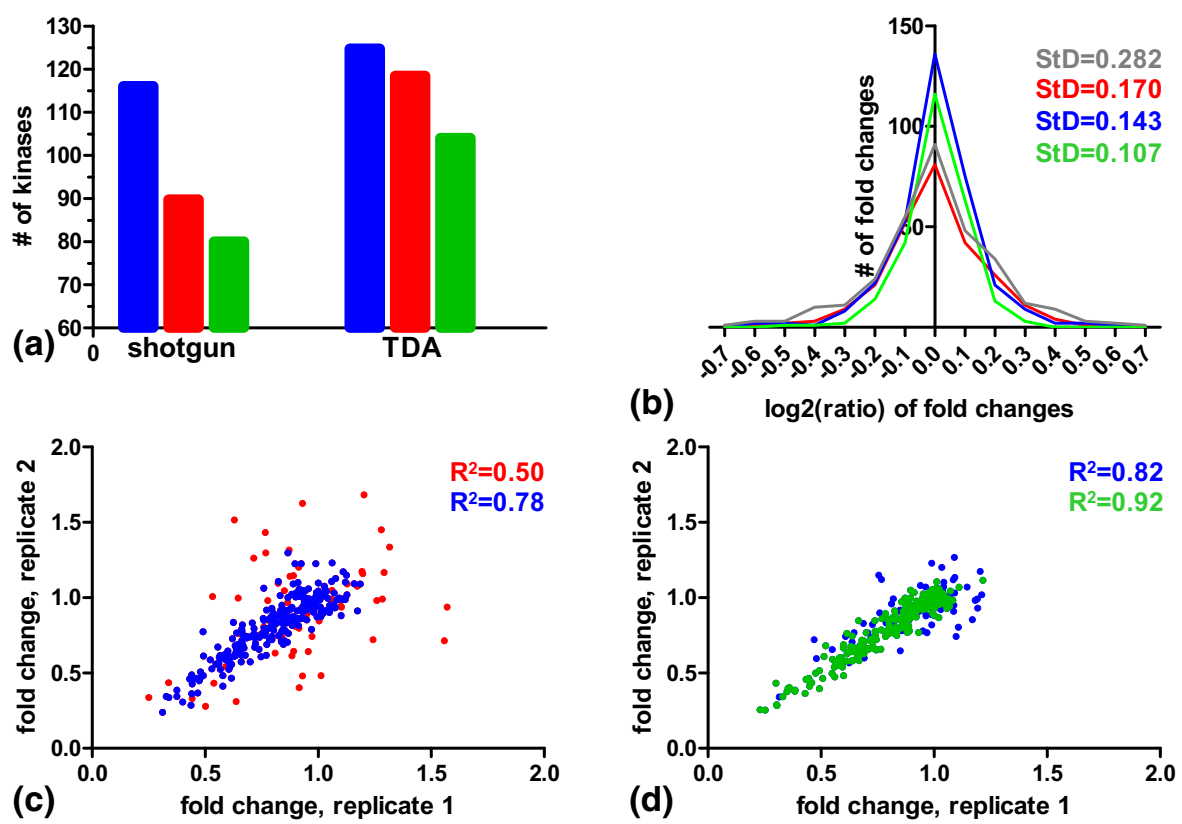

(c)

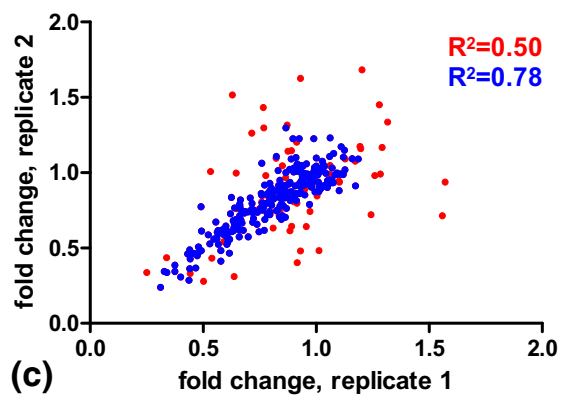

Figure 5. (a) Kinase statistics for seven shotgun and seven TDA runs. Blue bars: identified kinases, red bars: quantified kinases with $<3$ spectra, green bars: kinases reproduced in all seven experiments. (b) The distributions of the $\log _{2}$ ratios of the fold changes, grey: shotgun PQD with $>0$ spectra, red: shotgun PQD with four or more spectra, blue: targeted analysis PQD, green: targeted analysis PQD, only kinases that were reproduced in shotgun PQD. (c) Kinase fold change comparison of two repeat shotgun PQD experiments, 107 reproduced kinases with in total, red and blue, 85 reproduced kinases with four or more spectra, marked in blue. (d) Kinase fold change comparison of two repeat targeted analysis PQD experiments, 112 reproduced kinases with $>3$ spectra blue and green, the 85 kinases with four or more spectra that were reproduced in the shotgun experiment are marked in green. 
cation values determined for the 85 kinases quantified in both experiments from at least four spectra reproduced substantially better (the $>4$ spectra requirement for quantifying a protein is used in all our experiments). For these 85 kinases, the $R^{2}$ value was 0.78 and the standard deviation of the $\log _{2}$ ratios of the relative abundancies was 0.170 (cf. Figure 5b). Next we compared two replicate TDA experiments. In total 118 (11 more than in shotgun) kinases were reproduced. Amongst these, only six kinases were quantified with less than four spectra. It should be noted that quantification of these six kinases reproduced poorly just like in the shotgun experiments (data not shown). A total of 112 (27 more than in shotgun) kinases quantified with four or more quantified spectra were reproduced (Figure $5 \mathrm{~d}$ ). The $\mathrm{R}^{2}$ value was 0.82 and standard deviation of the $\log _{2}$ ratios was 0.143 . The relative abundancies of the 85 kinases that were reproduced in the shotgun experiments (marked in green in Figure $5 \mathrm{~d}$ ) had a $\mathrm{R}^{2}$ value of 0.92 and a standard deviation of 0.107 (Figure $5 b)$, hence the quantification precision for these kinases increased by more than 1.5-fold. The increase in kinase identification in targeted analysis experiments compared with shotgun experiments was due to the targeting of kinase-specific peptides on the inclusion list. The data presented here clearly indicate that repeated acquisition of tandem MS spectra for a given peptide improves precision of quantification to a similar degree as fragmentation and reporter ion based quantification of additional different peptides. This explains the overall increase in precision of quantification observed when the kinase enriched samples are analyzed using targeted data acquisition. The pairwise comparisons of the other experiments yielded very similar results.

\section{CID/HCD Based iTRAQ Quantification Compared with $P Q D$}

Recent versions of Orbitrap mass spectrometers have been equipped with additional collision cells enabling more triple-quadrupole-like fragmentation termed HCD, thus omitting the low-mass cut-off generally observed for resonance induced fragmentation on ion trap instruments [25]. In a recent study using an OrbitrapXL equipped with a octopole collision cell it was shown that HCD provided more precise iTRAQ reporter ion based quantification than PQD on the spectra level, but in a shotgun application was significantly slower than PQD, which resulted in a lower number of acquired spectra, fewer identified proteins and, overall, in a very similar quantification precision as PQD on the protein level [15]. Recently, Kocher and coworkers reported on the combination of ion trap CID and collision cell based HCD for sensitive and precise identification and quantification of iTRAQ labeled samples [12]. In this approach, the CID spectra were used for spectrum to sequence matching whereas HCD spectra were acquired at elevated collision energies to achieve high yields of reporter ions facilitating precise quantification. We have applied this approach for targeted detection of kinase proteins in our proteomic kinase assay, thereby evaluating if the required acquisition of two spectra (CID and HCD) for each inclusion list peptide would have any impact on kinase coverage.

In a first step, we optimized normalized collision energy (NCE) for HCD so that as abundant as possible reporter ions would be generated. Four targeted analysis experiments using the CID/HCD setup were performed with an inclusion list of 1534 peptides at four different NCEs, 40\%, 50\%, 60\%, 70\%. The average reporter ion area was calculated for the identified peptides separated into three groups according to their charge (Figure 6a). In agreement with Zhang et al. [26], we observed that peptides in different charge states have different NCE optima. The optimal values for doubly charged ions were between $50 \%$ and $60 \%$ NCE, while for $3+$ and $4+$ ions, a NCE of $70 \%$ was the best of those tested. For $3+$ the optimal NCE is likely to be at around $80 \%$ and for $4+$ at even higher values. In the 80 shotgun analyses acquired on kinase enriched samples, doubly charged ions are the most predominant species. Consequently, the majority of inclusion list peptides were targeted as doubly charged species (67\%) followed by $3+, 23 \%$ and $4+$ are rare with $3 \%$. We chose the $70 \%$ NCE value as a compromise, since it is located between the best values for $2+$ and $3+$ and the average reporter ion area for $2+$ drops by only $10 \%$ compared with the $60 \mathrm{NCE}$ value. It is worth noting here that the instrument method allows for selection of individual NCEs for different peptides on the inclusion list. Unfortunately, it is not possible to also set different NCEs for CID and HCD, respectively. A potential solution to this problem could be the use of an AMT strategy [21, 22] for identifying the inclusion list peptides, which would then eliminate the need for performing CID.

Two replicate TDA experiments using the CID/HCD approach were performed on an Orbitrap XL and compared with targeted analysis experiments using PQD acquired on the same instrument. One hundred twenty-four kinases quantified with at least four spectra were reproduced (Figure 6b), (see supplementary Table 3). Correlation of quantification results between the two CID/HCD experiments was excellent yielding an $\mathrm{R}^{2}$ value of 0.96 and $\log _{2}$ standard deviation of 0.064 (Figure 6c). When considering only the 110 protein kinases that were also reproducibly quantified with at least 4 spectra in the back-to-back PQD experiments (fold changes marked in blue in Figure 6b) $R^{2}$ was as high as 0.97 and a standard deviation of 0.054 was achieved (Figure 6c). This demonstrates an $\sim 2$-fold higher quantification precision for the CID/HCD approach compared with PQD. The higher number of identified and quantified kinases obtained from targeted analysis experiments with the CID/HCD setup (12 additional kinases) is likely due to the in average better quality of spectra acquired with CID as compared with PQD enabling improved protein identification. 

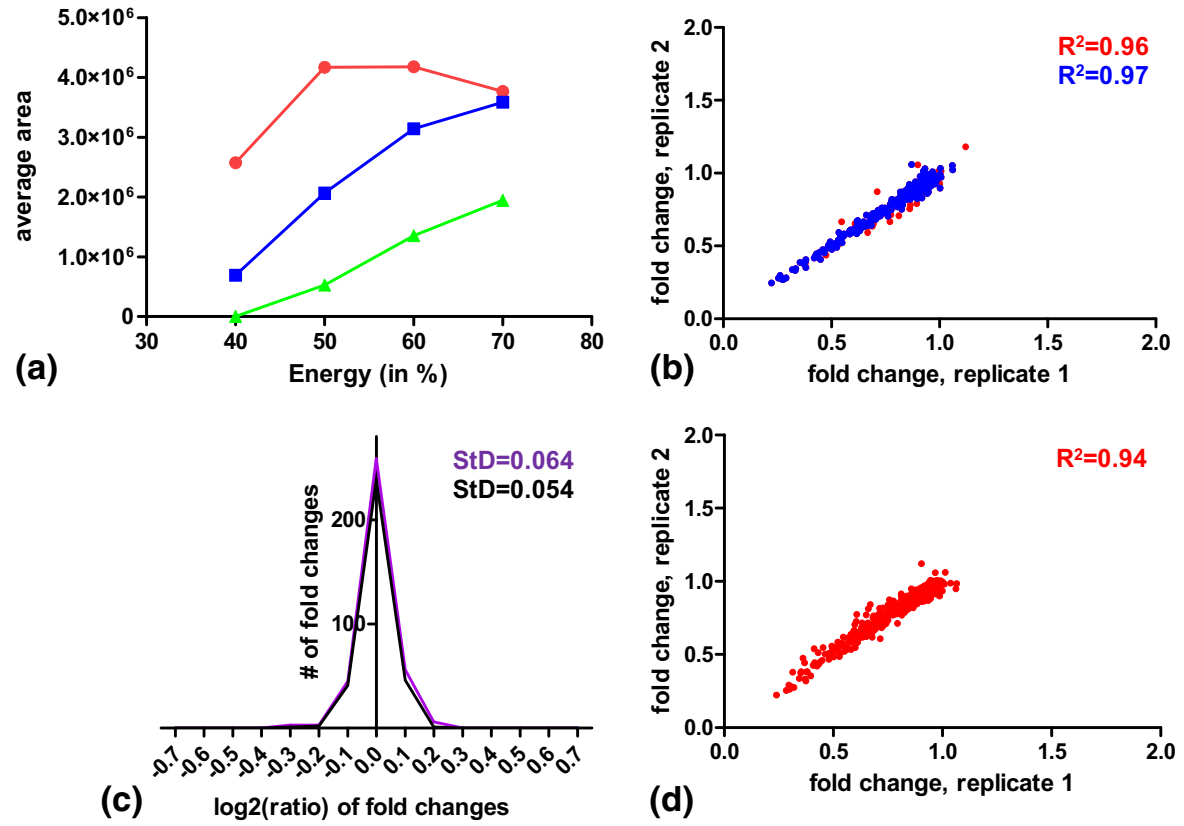

Figure 6. (a) Average reporter ion area for peptides in different charge states, red $2+$, blue $3+$, and green $4+$, calculated for four experiments with different HCD collision energies, $40 \%, 50 \%$, $60 \%$, and $70 \%$. (b) Kinase fold change comparison of two repeat TDA CID/HCD experiments, 124 reproduced kinases with four or more spectra are marked in red and blue, the 110 kinases with more than three spectra that were reproduced in the targeted analysis PQD experiment are marked in blue. (c) The distributions of the $\log _{2}$ ratios of the fold changes, violet: TDA CID/HCD mode, black: TDA $\mathrm{CID} / \mathrm{HCD}$ mode only kinases that were reproduced in TDA PQD experiments. (d) Kinase fold change comparison of two CID/HCD experiments performed on the same sample but on two different Orbitraps with two different LC systems. Fold changes for 123 reproduced kinases with four or more spectra are compared.

The precision increase of kinase quantification from TDA experiments with the PQD setup to TDA experiments with the CID/HCD setup can mainly be attributed to the better ion statistics for reporter ions in the MS/MS spectra performed in HCD mode. These observations agree with Kocher et al. [12].

\section{Inter-Instrument Reproducibility}

High reproducibility in repeat back-to-back experiments on the same mass spectrometer is a good measure for precision. However, a quantification based proteomic assay should ideally reproduce equally well when the sample is applied on different mass spectrometers, thus excluding a mass spectrometer specific bias. To assess inter-instrument reproducibility of the quantitative readout, we analyzed the iTRAQ reference sample on two similar but slightly different Orbitrap mass spectrometers (Orbitrap XL and the newer Orbitrap Velos) that were coupled to different Eksigent NanoLCs using the targeted analysis method with the $\mathrm{CID} / \mathrm{HCD}$ setup. One hundred twenty-three kinases with at least four spectra were reproduced, and their measured relative abundancies compared (Figure 6d); the $R^{2}$ value was 0.94 and the standard deviation of the $\log _{2}$ ratios of the determined fold changes was 0.090 . These values were comparable to those obtained com- paring two back-to-back runs on the same mass spectrometer $/ \mathrm{LC}$ system, $\left(\mathrm{R}^{2}=0.96, \mathrm{StD}=0.064\right)$. The slope of the fitted line was 0.95 and the intercept was 0.05 . This indicates a very low mass spectrometer/LC system specific bias, which in turn is indicative of not only high precision, but also of good accuracy.

\section{Conclusions}

We have developed a targeted mass spectrometric data acquisition methodology that is useful in applications where it is essential to re-identify and re-quantify a defined set of target proteins in a complex sample. We have successfully applied this strategy to the quantitative detection of a set of more than 100 protein kinases in an affinity-captured subproteome. The targeted approach outperforms the standard shotgun approach in terms of identification numbers and quantification precision. In addition, it is shown that the targeted data acquisition approach is compatible with a dual peptide fragmentation strategy using CID for generation of backbone fragments, facilitating peptide identifications and HCD for improved reporter ion based quantification. This approach yielded a higher identification rate and more precise quantification compared with targeted peptide fragmentation using pulsed-Q-dissociation. Considering the increasing amount of data stored in public 
data repositories [27, 28], and the improving tools for prediction of proteotypic peptides $[29,30]$, we anticipate that generation of appropriate inclusion mass lists for any given sample will be largely simplified, requiring acquisition of less experimental data.

\section{Acknowledgments}

The authors acknowledge support for this study by the Bundesministerium fur Bildung und Forschung, Spitzencluster BioRN, Verbundprojekt Inkubator, BioRN-INE-TP01 and BioRNIND-TP02. The authors thank Frank Weisbrodt for help with preparing the figures and Ulrich Kruse and Gerard Drewes for helpful discussions.

\section{Appendix A Supplementary Material}

Supplementary material associated with this article may be found in the online version at doi:10.1016/ j.jasms.2010.01.012.

\section{References}

1. Peng, J.; Schwartz, D.; Elias, J. E.; Thoreen, C. C.; Cheng, D.; Marsischky, G.; Roelofs, J.; Finley, D.; Gygi, S. P. A Proteomics Approach to Understanding Protein Ubiquitination. Nat. Biotechnol. 2003, 21, 921926.

2. Steen, H.; Kuster, B.; Fernandez, M.; Pandey, A.; Mann, M. Tyrosine Phosphorylation Mapping of the Epidermal Growth Factor Receptor Signaling Pathway. J. Biol. Chem. 2002, 277, 1031-1039.

3. Bantscheff, M.; Scholten, A.; Heck, A. J. Revealing Promiscuous DrugTarget Interactions by Chemical Proteomics. Drug Discov. Today 2009, 14 (21-22), 1021-1029.

4. Lolli, G.; Thaler, F.; Valsasina, B.; Roletto, F.; Knapp, S.; Uggeri, M.; Bachi, A.; Matafora, V.; Storici, P.; Stewart, A.; Kalisz, H. M.; Isacchi, A. Inhibitor Affinity Chromatography: Profiling the Specific Reactivity of the Proteome with Immobilized Molecules. Proteomics 2003, 3, 12871298.

5. Saxena, C.; Higgs, R. E.; Zhen, E.; Hale, J. E. Small-Molecule Affinity Chromatography Coupled Mass Spectrometry for Drug Target Deconvolution. Expert Opin. Drug Discov. 2009, 4, 701-714.

6. Bantscheff, M.; Eberhard, D.; Abraham, Y.; Bastuck, S.; Boesche, M.; Hobson, S.; Mathieson, T.; Perrin, J.; Raida, M.; Rau, C.; Reader, V.; Sweetman, G.; Bauer, A.; Bouwmeester, T.; Hopf, C.; Kruse, U.; Neubauer, G.; Ramsden, N.; Rick, J.; Kuster, B.; Drewes, G. Quantitative Chemical Proteomics Reveals Mechanisms of Action of Clinical ABL Kinase Inhibitors. Nat. Biotechnol. 2007, 25, 1035-1044.

7. Cravatt, B. F.; Wright, A. T.; Kozarich, J. W. Activity-Based Protein Profiling: From Enzyme Chemistry to Proteomic Chemistry. Annu. Rev. Biochem. 2008, 77, 383-414.

8. Oppermann, F. S.; Gnad, F.; Olsen, J. V.; Hornberger, R.; Greff, Z.; Keri, G.; Mann, M.; Daub, H. Large-Scale Proteomics Analysis of the Human Kinome. Mol. Cell. Proteom. 2009, 8, 1751-1764.

9. Patricelli, M. P.; Szardenings, A. K.; Liyanage, M.; Nomanbhoy, T. K.; Wu, M.; Weissig, H.; Aban, A.; Chun, D.; Tanner, S.; Kozarich, J. W. Functional Interrogation of the Kinome Using Nucleotide Acyl Phosphates. Biochemistry 2007, 46, 350-358.

10. Wissing, J.; Jansch, L.; Nimtz, M.; Dieterich, G.; Hornberger, R.; Keri, G.; Wehland, J.; Daub, H. Proteomics Analysis of Protein Kinases by Target
Class-Selective Prefractionation and Tandem Mass Spectrometry. Mol. Cell. Proteom. 2007, 6, 537-547.

11. Oeljeklaus, S.; Meyer, H. E.; Warscheid, B. New Dimensions in the Study of Protein Complexes Using Quantitative Mass Spectrometry. FEBS Lett. 2009, 583, 1674-1683.

12. Kocher, T.; Pichler, P.; Schutzbier, M.; Stingl, C.; Kaul, A.; Teucher, N.; Hasenfuss, G.; Penninger, J. M.; Mechtler, K. High Precision Quantitative Proteomics Using iTRAQ on an LTQ Orbitrap: A New Mass Spectrometric Method Combining the Benefits of All. J. Proteome Res. 2009, 8, 4743-4752.

13. Olsen, J. V.; Schwartz, J. C.; Griep-Raming, J.; Nielsen, M. L.; Damoc, E.; Denisov, E..; Lange, O.; Remes, P.; Taylor, D.; Splendore, M.; Wouters, E. R.; Senko, M.; Makarov, A.; Mann, M.; Horning, S.; A Dual Pressure Linear Ion Trap-Orbitrap Instrument with Very High Sequencing Speed. Mol. Cell. Proteom. 2009, 8(12), 2759-2769.

14. Olsen, J. V.; de Godoy, L. M.; Li, G.; Macek, B.; Mortensen, P.; Pesch, R. Makarov, A.; Lange, O.; Horning, S.; Mann, M. Parts Per Million Mass Accuracy on an Orbitrap Mass Spectrometer Via Lock Mass Injection into a C-Trap. Mol. Cell. Proteom. 2005, 4, 2010-2021.

15. Bantscheff, M.; Boesche, M.; Eberhard, D.; Matthieson, T.; Sweetman, G.; Kuster, B. Robust and Sensitive iTRAQ Quantification on an LTQ Orbitrap Mass Spectrometer. Mol. Cell. Proteom. 2008, 7, 1702-1713.

16. Perkins, D. N.; Pappin, D. J.; Creasy, D. M.; Cottrell, J. S. ProbabilityBased Protein Identification by Searching Sequence Databases Using Mass Spectrometry data. Electrophoresis 1999, 20, 3551-3567.

17. Peng, J.; Elias, J. E.; Thoreen, C. C.; Licklider, L. J.; Gygi, S. P. Evaluation of Multidimensional Chromatography Coupled with Tandem Mass Spectrometry (LC/LC-MS/MS) for Large-Scale Protein Analysis: The Yeast proteome. J. Proteome Res. 2003, 2, 43-50.

18. Elias, J. E.; Gygi, S. P. Target-Decoy Search Strategy for Increased Confidence in Large-Scale Protein Identifications by Mass Spectrometry. Nat. Methods 2007, 4, 207-214.

19. Hanke, S.; Besir, H.; Oesterhelt, D.; Mann, M. Absolute SILAC for Accurate Quantitation of Proteins in Complex Mixtures Down to the Attomole Level. J. Proteome Res. 2008, 7, 1118-1130.

20. Jaffe, J. D.; Keshishian, H.; Chang, B.; Addona, T. A.; Gillette, M. A.; Carr, S. A. Accurate Inclusion Mass Screening: A Bridge from Unbiased Discovery to Targeted Assay Development for Biomarker Verification. Mol. Cell. Proteom. 2008, 7, 1952-1962.

21. Jaitly, N.; Monroe, M. E.; Petyuk, V. A.; Clauss, T. R.; Adkins, J. N.; Smith, R. D. Robust Algorithm for Alignment of Liquid Chromatography-Mass Spectrometry Analyses in an Accurate Mass and Time Tag Data Analysis Pipeline. Anal. Chem. 2006, 78, 7397-7409.

22. Zimmer, J. S.; Monroe, M. E.; Qian, W. J.; Smith, R. D. Advances in Proteomics Data Analysis and Display Using an Accurate Mass and Time Tag approach. Mass Spectrom. Rev. 2006, 25, 450-482.

23. Bantscheff, M.; Schirle, M.; Sweetman, G.; Rick, J.; Kuster, B. Quantitative Mass Spectrometry in Proteomics: A Critical Review. Anal. Bioanal. Chem. 2007, 389, 1017-1031.

24. Ow, S. Y.; Salim, M.; Noirel, J.; Evans, C.; Rehman, I.; Wright, P. C. iTRAQ Underestimation in Simple and Complex Mixtures: The good, the Bad, and the Ugly. J. Proteome Res. 2009, 8(11), 5347-5355.

25. Olsen, J. V.; Macek, B.; Lange, O.; Makarov, A.; Horning, S.; Mann, M. Higher-Energy C-Trap Dissociation for Peptide Modification Analysis. Nat. Methods 2007, 4, 709-712.

26. Zhang, Y.; Ficarro, S. B.; Li, S.; Marto, J. A. Optimized Orbitrap HCD for Quantitative Analysis of Phosphopeptides. J. Am. Soc. Mass Spectrom. 2009, 20, 1425-1434.

27. Desiere, F.; Deutsch, E. W.; King, N. L.; Nesvizhskii, A. I.; Mallick, P. Eng, J.; Chen, S.; Eddes, J.; Loevenich, S. N.; Aebersold, R. The PeptideAtlas Project. Nucleic Acids Res. 2006, 34, D655-658.

28. Deutsch, E. W.; Lam, H.; Aebersold, R. PeptideAtlas: A Resource for Target Selection for Emerging Targeted Proteomics Workflows. EMBO Rep. 2008, 9, 429-434.

29. Fusaro, V. A.; Mani, D. R.; Mesirov, J. P.; Carr, S. A. Prediction of High-Responding Peptides for Targeted Protein Assays by Mass Spectrometry. Nat. Biotechnol. 2009, 27, 190-198.

30. Mallick, P.; Schirle, M.; Chen, S. S.; Flory, M. R.; Lee, H.; Martin, D. Ranish, J.; Raught, B.; Schmitt, R.; Werner, T.; Kuster, B.; Aebersold, R. Computational Prediction of Proteotypic Peptides for Quantitative Proteomics. Nat. Biotechnol. 2007, 25, 125-131. 